\title{
Families of Galois closure curves for plane quartic curves
}

\author{
By
}

\author{
Hisao YoshiHaRa
}

\begin{abstract}
For a smooth quartic plane curve $C$ we show an existence of a family of Galois closure curves $\varphi: S \longrightarrow C$, where $S$ is a nonsingular projective surface and $\varphi^{-1}(P)$ is isomorphic to the Galois closure curve $C_{P}$ for a general point $P \in C$. Moreover we determine the types of singular fibers. As a corollary we can say that $C_{P}$ is not isomorphic to $C_{Q}$ if $P$ is close to $Q$.
\end{abstract}

\section{Introduction}

Let the ground field of our discussions be the complex number field. Let $C$ be a smooth curve of degree $d(\geq 4)$ in the projective plane $\mathbb{P}^{2}$ and $P$ be a point in $C$. Then we have a projection $\pi_{P}: C \longrightarrow l$ with center $P$, where $l$ is a line not passing through $P$. This projection induces the extension of fields $\pi_{P}{ }^{*}: k(l) \hookrightarrow k(C)=K$ with $[K: k(l)]=d-1$. The structure of this extension does not depend on the choices of $l$, but on $P$. So we denote $k(l)$ by $K_{P}$. There rise several questions under this situation. We have studied them from several points of view, see [4], [8] and also [9]. Especially we have considered the following: let $L_{P}$ be the Galois closure of this extension $K / K_{P}$.

Definition. Let $C_{P}$ be the nonsingular projective model of $L_{P}$. We call $C_{P}$ the Galois closure curve at $P \in C$ and let $g(P)$ be the genus of $C_{P}$. In the case where $K / K_{P}$ is a Galois extension, we call $P$ a Galois point for $C$.

We have studied the Galois group $G_{P}:=\operatorname{Gal}\left(L_{P} / K_{P}\right)$, the genus $g(P)$ and the number of Galois points etc. (cf. [8])

The motive for this research is the interest to know how the curve $C_{P}$ varies when $P$ moves on $C$. In the case where $d=4$ we can answer the problem raised from this motive. We can construct a family $\varphi: S \longrightarrow C$ satisfying that $\varphi^{-1}(P) \cong C_{P}$ for a general point $P \in C$ and study the structure of this fiber space. Then we consider whether $C_{P}$ is not isomorphic to $C_{Q}$ if $P \neq Q$.

Received November 25, 2002

Revised May 6, 2003 


\section{Statement of results}

Unless otherwise mentioned, we assume that $C$ is a smooth quartic curve. We have found several facts by the study under the above frame work, see [4]. Especially the Galois group $G_{P}$ is isomorphic to the symmetric group of degree three if $P$ is a general point, and there exist at most four Galois points.

Let $T_{P}$ denote the tangent line to $C$ at $P$ and $I\left(C_{1}, C_{2} ; Q\right)$ denote the intersection number of $C_{1}$ and $C_{2}$ at $Q$. The point $P \in C$ is said to be an $i$-flex $(i=1,2)$, where $i=I\left(C, T_{P} ; P\right)-2$. We have the fact $\sum_{Q \in C}\left\{I\left(C, T_{Q} ; C\right)-\right.$ $2\}=24$ (cf. [3]).

Note that a Galois point is a 2 -flex (but not the converse). Since the center of the projection is in $C$, we make the following definition. Let $\nu_{i}=\nu_{i}(P)$, where $i=1,2$, be the number of lines $l$ satisfying that $l \ni P$ and $I(C, l ; Q)=$ $i+1$ for some $Q \in C$, where $Q \neq P$. Then put $m=\nu_{1}$ [resp. $\left.\nu_{1}+1\right]$ if $P$ is not a flex [resp. is a 1-flex], and $n=\nu_{2}$ [resp. $\nu_{2}+1$ ] if $P$ is a 1-flex [resp. 2-flex]. Applying the Riemann-Hurwitz formula to the covering $\pi_{P}: C \longrightarrow l$, we get $m+2 n=10$, moreover we have $g(P)=10-n$ if $P$ is not a Galois point, where $0 \leq n \leq 4$ (cf. [4]). Let $\Sigma$ be the set of points $P$ in $C$ satisfying the following condition (1) or (2):

(1) $P$ is a 2 -flex.

(2) $Q(\neq P)$ is a 1 -flex and $T_{Q} \ni P$.

Clearly the number of elements of $\Sigma$ is at most 24 .

Let $\left(D_{1}, D_{2}\right)$ [resp. $\left.D^{2}\right]$ denote the intersection number of $D_{1}$ and $D_{2}$ [resp. the self-intersection number of $D$ ] on some surface.

Under these situations our main theorem can be stated as follows:

Theorem 2.1. $\quad$ There exist a nonsingular projective minimal surface of general type $S$ and a morphism $\varphi: S \longrightarrow C$ with the following properties:

(1) If $P \in C \backslash \Sigma$, then $\varphi^{*}(P) \cong C_{P}$ and $g(P)=10$.

(2) If $P \in \Sigma$, then the assertions are stated in three sub cases separately.

(2-1) If $P$ is not a 2-flex, then $\varphi^{*}(P)$ is an irreducible curve with n-pieces of nodes and $g(P)=10-n$.

(2-2) If $P$ is a 2-flex but not a Galois point, then $\varphi^{*}(P)=\Delta+E$ as divisors, where $\Delta$ and $E$ are irreducible curves with the following properties:

(i) $g(\Delta)=10-n$ and $\Delta$ has $(n-1)$-pieces of nodes.

(ii) $\Delta^{2}=-2$ and $E$ is a (-2)-curve.

(iii) $\Delta$ and $E$ meet at in two points, where they have normal crossings.

(2-3) If $P$ is a Galois point, then $\varphi^{*}(P)=C_{1}+C_{2}+E$ as divisors with the following properties:

(i) $C_{i} \cong C(i=1,2),\left(C_{i}\right)^{2}=-5$ and $E$ is a $(-2)$-curve.

(ii) $\varphi^{*}(P)$ is a divisor with normal crossings.

(iii) $\left(C_{1}, C_{2}\right)=4,\left(C_{i}, E\right)=1(i=1,2)$. 


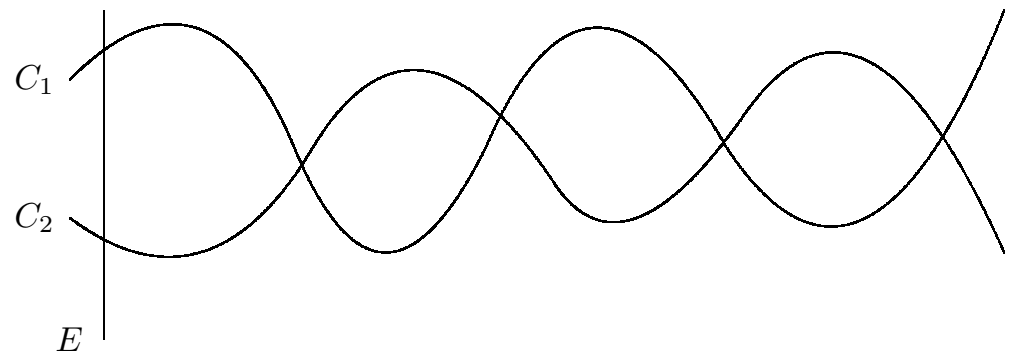

Especially, if $C$ is a general quartic curve, then the cardinality of $\Sigma$ is 24, and for the point $P \in \Sigma, \varphi^{*}(P)$ is an irreducible curve with one node and its genus is 9 . Furthermore the canonical bundle of $S$ is ample.

As a corollary we have the following assertion which has a close relation to the one in [5, Theorem 2.3.1]

Corollary 2.1. For any $P \in C$, there exists an open neighborhood $U_{P}$ of $P$ satisfying that $C_{Q}$ is not isomorphic to $C_{Q^{\prime}}$ if $Q$ and $Q^{\prime}$ belong to $U_{P}$ and $Q \neq Q^{\prime}$.

This assertion does not necessarily hold true if $Q^{\prime}$ is not near to $Q$. Indeed, we have the following.

Remark 1. Suppose $C$ has a non-trivial automorphism $\sigma$. Then, it is a restriction of a projective transformation. It is easy to see that $C_{Q} \cong C_{\sigma(Q)}$, however $\sigma(Q) \neq Q$ for a general point $Q$.

Remark 2. We mention a uniqueness of $S$ in the following sense: let $\varphi^{\prime}: S^{\prime} \longrightarrow C$ be a surjective morphism satisfying that $S^{\prime}$ is a smooth projective minimal algebraic surface and $\varphi^{\prime-1}(P) \cong C_{P}$ for a general point $P \in C$. Suppose that $\varphi^{\prime}$ is induced from the projection $\pi_{P}$, i.e., $S^{\prime}$ is the nonsingular projective minimal model of the minimal splitting variety (see [7], for the definition) for the triple covering $p_{1}: C \times C \longrightarrow C \times \mathbb{P}^{1}$, where $p_{1}(P, Q)=\left(P, \pi_{P}(Q)\right)$. Then, there exists an isomorphism $\psi: S^{\prime} \longrightarrow S$ satisfying that $\varphi \cdot \psi=\varphi^{\prime}$.

The author expresses his gratitude to T. Takahashi and H. Sakai for useful conversations and also to the referee who pointed out the error in the determination of the singular fiber.

\section{Proofs}

Let $(X: Y: Z)$ be a set of homogeneous coordinates on $\mathbb{P}^{2}$ and put $x=X / Z, y=Y / Z$. Let $C_{0}$ be the affine part $Z \neq 0$ of $C$ and $f(x, y)=0$ be the defining equation. In this first paragraph and two lemmas below, we do not assume that the degree $d$ of $C$ is four. Let $y=t(x-u)+v$ be a line passing through a point $P=(u, v) \in \mathbb{A}^{2}$ and let $D=D(t, u, v)$ be the discriminant of $f(x, t(x-u)+v)$ with respect to $x$. Then the degree of $D$ with respect to 
$t$ is $c=d(d-1)$, which is the class of $C$. Similarly the (total) degree of $D$ with respect to $u$ and $v$ is $c=d(d-1)$. Let $\Phi$ be the homogeneous equation of the dual curve $\widehat{C}$ of $C$ and put $D^{\prime}(t, u, v)=\Phi(t,-1,-u t+v)$. The following assertion is shown in [8, Lemma 3$]$.

Lemma 3.1. We have $D=D^{\prime}$.

Moreover, in case $P=(u, v)$ is in $C_{0}$, we let $\Psi(t, u, v)$ be the discriminant of $F(x, t, u, v):=f(x, t(x-u)+v) /(x-u)$ with respect to $x$. Define $\bar{f}(t, u, v)$ to be the value of $F(x, t, u, v)$ at $x=u$. Then we have the following.

Lemma 3.2. $\quad(-1)^{d-1}(\bar{f}(t, u, v))^{2} \Psi(t, u, v)=\Phi(t,-1,-u t+v)$.

Proof. The proof is done by direct computation of the discriminant and is essentially the same as in the proof of [8, Lemma 12].

Hereafter we assume that $d=4$. Let $B_{0}$ be the divisor defined by $\Psi$ in $\mathbb{A}^{1} \times C_{0}$. Take the other affine parts of $S_{0}:=\mathbb{P}^{1} \times C$ and define the similar divisor as $B_{0}$. Let $B$ be the divisor on $S_{0}$ obtained from patching such ones. It is easy to see that the support of $B \backslash B_{0}$ consists of finitely many points. Moreover, putting $t=T_{1} / T_{0}, u=U / W$ and $v=V / W$, we define $\widetilde{D}\left(T_{0}, T_{1} ; U, V, W\right):=$ $T_{0}^{12} W^{12} D(t, u, v)$.

Claim 1. The divisor $B$ is even, i.e., $\mathcal{O}_{S_{0}}(B)=\mathcal{L}^{\otimes 2}$ for some $\mathcal{L} \in$ $\operatorname{Pic}\left(S_{0}\right)$.

Proof. We can find a polynomial $G$ satisfying that $\widetilde{D} / G^{2}$ defines a rational function on $\mathbb{P}^{1} \times \mathbb{P}^{2}$ and the restriction of $\widetilde{D} / G^{2}$ to $\mathbb{P}^{1} \times C$ defines also a rational function on $\mathbb{P}^{1} \times C$. The right hand side of the equation in Lemma 3.2 is obtained by restricting $D$ to $\mathbb{A}^{1} \times C_{0}$. The same fact holds true on each affine part, hence we infer that $B$ is even.

Let $X_{1}$ be the affine surface defined by $f(u, v)=0$ and $F(x, t, u, v)=0$ in $\mathbb{A}^{2} \times \mathbb{A}^{2} \subset \mathbb{P}^{2} \times \mathbb{P}^{2}$. Let $\bar{X}_{1}$ be the projective closure of $X_{1}$ in $\mathbb{P}^{2} \times \mathbb{P}^{2}$. Then it is easy to see that the second projection induces a fiber space $\bar{X}_{1} \longrightarrow C$, each fiber of which is isomorphic to $C$. Hence $X_{1}$ is irreducible. Let $S_{1}$ be a nonsingular projective relative minimal surface which is birational to $X_{1}$.

Claim 2. $S_{1}$ is isomorphic to $C \times C$.

Proof. Since the function field $k\left(S_{1}\right)$ is equal to $k\left(X_{1}\right)=k(x, t, u, v)$, where $x, t, u$ and $v$ satisfy $f(u, v)=F(x, t, u, v)=0$ and $t=(y-v) /(x-u)$, we have $k(x, t, u, v)=k(x, y, u, v)$. Thus $S_{1}$ and $C \times C$ are birationally equivalent to each other. They are not ruled surfaces, hence they are isomorphic to each other.

Claim 3. There exists a finite morphism $p_{1}: S_{1} \longrightarrow S_{0}$ of degree three. 
Proof. Define $p_{1}: C \times C \longrightarrow l \times C \cong \mathbb{P}^{1} \times C$ by $p_{1}(Q, P)=\left(\pi_{P}(Q), P\right)$. Since $\pi_{P}: C \longrightarrow l$ is a triple covering for each $P$, the assertion is clear.

Claim 4. The divisor $B$ is reduced and its singularity is isomorphic to the one defined by $y^{2}+x^{3 k}=0$, where $k=1,2$.

Proof. Since $C$ has only a finitely many flexes, for a general $(a, b)=$ $(a: b: 1) \in C$ [resp. general $\left.\tau \in \mathbb{P}^{1}\right], \Psi(t, a, b)$ [resp. $\left.\Psi(\tau, u, v)\right]$ has no multiple factors. Therefore $B$ is reduced. Similarly we infer that the point $\left(t_{0}, u_{0}, v_{0}\right) \in B$ is a singular point if and only if $P=\left(u_{0}, v_{0}\right) \in C$ and the line $l_{0}: y=t_{0}\left(x-u_{0}\right)+v_{0}$ satisfy one of the following conditions:

(1) $l_{0}=T_{Q}$, where $Q \neq P$ and $Q$ is a flex of $C$.

(2) $l_{0}=T_{P}$ and $P$ is a 2 -flex.

We make use of Lemma 3.2 to determine the type of the singularity. The singularity is a local property, so we can assume as follows:

In the case (1), $P=(0,0), f(x, y)=x+a y+f_{2}+f_{3}+f_{4}$ and $f(x, 0)=$ $x(b x+1)^{3}$, where $f_{i}$ is a homogeneous polynomial of degree $i$ and $b \neq 0$.

In the case $(2), P=(0,0), f(x, y)=y+f_{2}+f_{3}+f_{4}$ and $f(x, 0)=c x^{4}$, where $c \neq 0$.

First we treat the case (1). We consider the singularity at $t=u=v=0$. By direct computation we have $\bar{f}(t, u, v)=1+a t+(*)$, where $(*)$ vanishes at $(0,0,0)$. Since $(-1 / b, 0)=(1: 0:-b)$ is a 1 -flex of $C$ and $Y=0$ is the tangent line to $C$ at the flex, we see that the dual curve $\widehat{C}$ of $C$ has a $(2,3)$-cusp at $(0: 1: 0) \in \widehat{\mathbb{P}^{2}}$ and the tangent line to $\widehat{C}$ at the cusp is given by $X-b Z=0$. Therefore, putting $\xi=X / Y, \eta=Z / Y$, we have the defining equation of $\widehat{C}$ is $\widehat{f}(\xi, \eta)=(\xi-b \eta)^{2}+\widehat{f}_{3}+\cdots+\widehat{f}_{12}$, where $\widehat{f}_{i}$ is the homogeneous polynomial of degree $i$. Since $(0,0) \in \widehat{C}$ is a $(2,3)$-cusp, if we put $\widehat{f}_{3}(\xi, \eta)=\sum_{i+j=3} a_{i j} \xi^{i} \eta^{j}$, then we have $\widehat{f}_{3}(b, 1)=\sum_{i+j=3} a_{i j} b^{i} \neq 0$. Thus we obtain $\Phi(X, Y, Z)=(X-b Z)^{2} Y^{10}+\widehat{f}_{3}(X, Z) Y^{9}+\cdots$. Near $(0,0)$ the curve $C$ can be expressed locally as $u=\phi(v)$, where $\phi=\phi(v)$ is a holomorphic function at $v=0$ and $\phi(0)=0$. Therefore we have $\Phi(t,-1,-\phi t+v)=$ $\{(t-b v)+b \phi t\}^{2}-\widehat{f}_{3}(t,-\phi t+v)+\cdots$. If we take a new system of coordinates $(s, v)$, where $s=t-b v$, this becomes

$$
\begin{gathered}
\{s+b \phi(s+b v)\}^{2}-\widehat{f}_{3}(s+b v,-\phi(s+b v)+v)+\cdots \\
=s^{2}-\sum_{i+j=3} a_{i j}(s+b v)^{i} v^{j}+(\text { higer terms }) .
\end{gathered}
$$

Since $\bar{f}(t, u, v)$ is a unit in the convergent power series ring $\mathbb{C}\{t, v\}$ and $\sum_{i+j=3} a_{i j} b^{i} \neq 0$, this implies that the singularity is the $(2,3)$-cusp.

Next we treat the case (2). We consider the singularity at $t=u=v=0$. Since $f(x, y)$ can be expressed as $y+c x^{4}+g(x, y) y$, where $c \neq 0$ and $g(0,0)=0$, we have $\bar{f}(t, u, v)=t+4 c u^{3}+t g(u, v)+\bar{g}(t, u, v) v$, where $\bar{g}(t, u, v)$ is the value of $\{g(x, t(x-u)+v)-g(u, v)\} /(x-u)$ at $x=u$. Since $(0: 0: 1)$ is a 2 -flex of $C$ and $Y=0$ is the tangent line to $C$ at the flex, the dual curve $\widehat{C}$ has a 
$(3,4)$-cusp at $(0: 1: 0) \in \widehat{\mathbb{P}^{2}}$ and the tangent line to $\widehat{C}$ at the cusp is given by $Z=0$. Therefore, putting $\xi=X / Y, \eta=Z / Y$, we have the defining equation of $\widehat{C}$ is $\widehat{f}(\xi, \eta)=\eta^{3}+\widehat{f}_{4}+\cdots+\widehat{f}_{12}$, where $\widehat{f}_{4}(\xi, 0)=a \xi^{4} \neq 0$. Thus we obtain $\Phi(X, Y, Z)=Y^{9} Z^{3}+\widehat{f}_{4}(X, Z) Y^{8}+\cdots$. Near $(0,0)$ the curve $C$ can be expressed locally as $v=\phi(u)=-c u^{4}+\cdots$, where $\phi=\phi(u)$ is a holomorphic function at $u=0$ and $c \neq 0$. Therefore we have $\Phi(t,-1,-u t+v)=-(-u t+\phi)^{3}+$ $\widehat{f}_{4}(t,-u t+\phi)+\cdots$, which is equal to $-\left\{t+4 c u^{3}+t g(u, v)+\bar{g}(t, u, v) \phi\right\}^{2} \Psi(t, u, v)$. Since $\widehat{f}_{4}(t,-u t+\phi)=a t^{4}+\cdots(a \neq 0)$, the multiplicity of the singular point is two. Perform the quadratic transformation $t=s u, u=u$. After dividing by $u^{2}$ both sides, we obtain $-u^{4}\left(-s+\phi_{2}\right)^{3}+u^{2} \widehat{f}_{4}\left(s,-u t+\phi_{3}\right)+\cdots=-(s+$ $\left.4 c u^{2}+s g(u, \phi)+\bar{g} \phi\right)^{2} \Psi(s u, u, \phi(u))$, where we put $\phi_{2}=\phi / u^{2}$ and $\phi_{3}=\phi / u$. Therefore $\Psi(s u, u, \phi)$ is divisible by $u^{2}$. Put $\Psi_{1}(s, u)=\Psi(s u, u, \phi) / u^{2}$. Then, we get $-u^{2}\left(-s+\phi_{2}\right)^{3}+\widehat{f}_{4}\left(s,-u s+\phi_{3}\right)+\cdots=-\left(s+4 c u^{2}+s g+\bar{g} \phi_{3}\right)^{2} \Psi_{1}(s, u)$. Once more perform the quadratic transformation $s=r u, u=u$. After dividing by $u^{2}$ both sides, we obtain

$$
-u^{3}\left(-r+\phi_{1}\right)^{3}+u^{2} \widehat{f}_{4}\left(r,-u r+\phi_{2}\right)+\cdots=-\left(r+4 c u+r g+\bar{g} \phi_{2}\right)^{2} \Psi_{1}(r u, u),
$$

where $\phi_{1}=\phi(u) / u^{3}$. Thus $\Psi_{1}(r u, u)$ is divisible by $u^{2}$. Putting $\Psi_{2}(r, u)=$ $\Psi_{1}(r u, u) / u^{2}$, we obtain $-u\left(-r+\phi_{1}\right)^{3}+\widehat{f}_{4}\left(r,-u r+\phi_{2}\right)+\cdots=-(r+4 c u+$ $\left.r g+\bar{g} \phi_{2}\right)^{2} \Psi_{2}(r, u)$. Let $Q(r, u)$ be the leading form of $\Psi_{2}(r, u)$, i.e., the order two part of $\Psi_{2}(r, u)$. Then we have $u(r+c u)^{3}+a r^{4}=-(r+4 c u)^{2} Q$. This implies $a c=3^{3} / 4^{4}$. Therefore we obtain $Q=a r^{2}+(5 / 32) u r+(1 / 16) c u^{2}$, which has distinct factors. This implies that the singularity is of $A_{5}$-type.

Remark 3. If $C$ is a general quartic, then the divisor $B$ is irreducible.

Proof. Suppose that $B$ has an irreducible decomposition $B=B_{1}+\cdots+$ $B_{r}$. Then, each $B_{i}$ is an ample divisor, because $B_{i}$ is not a fiber of the projection $\pi_{1}: S_{0} \longrightarrow \mathbb{P}^{1}$ nor the one $\pi_{2}: S_{0} \longrightarrow C$. Hence $B$ is connected. Since the singularities of $B$ are only $(2,3)$-cusps, $B$ cannot be reducible.

Following Definitions 1.1 and 1.2 in [7], we define $S_{2}$ to be the discriminant variety of $S_{0}$. By Claim 1 we see $S_{2}$ is the double covering of $S_{0}$ branched along $B$. Let $S_{3}$ be the "minimal splitting variety" of $S_{1}$, then we have the following commutative diagram:

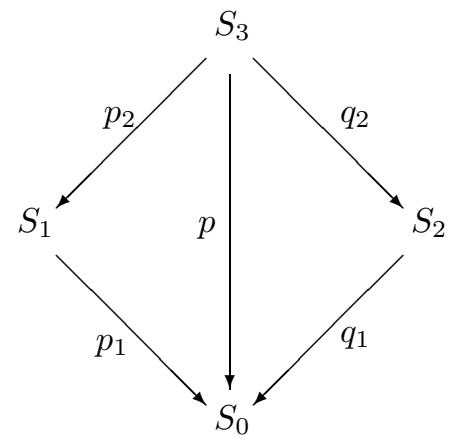


Clearly $S_{3}$ is a surface of general type. Note that these are objects over $C$. Let $\varphi_{i}: S_{i} \longrightarrow C$ be the structure morphism $(0 \leq i \leq 3)$. It is easy to see that if $P \in C \backslash \Sigma$, then $\varphi_{3}{ }^{*}(P)$ is "the minimal splitting variety" of $\pi_{P}: C \longrightarrow l$, i.e., it is the Galois closure curve $C_{P}$.

Claim 5. There exists an automorphism $\sigma$ on $S_{3}$ satisfying that $S_{3} /\langle\sigma\rangle$ $\cong S_{2}$. Moreover it has fixed points which are just $q_{2}^{-1}\left(\operatorname{Sing}\left(S_{2}\right)\right)$, where $\operatorname{Sing}\left(S_{2}\right)$ denotes the set of singular points of $S_{2}$.

Proof. The proofs of the former and latter statements are the same as in the one of [7, Proposition 1.3] and [7, Proposition 3.4] respectively.

By Claim 4 the singularities of $S_{2}$ are of type $A_{3 k-1}$, where $k=1$ or 2 . By Claim 5 we see that if $R_{0}$ is a singular point on $S_{2}$ of type $A_{1}$, then $q_{2}^{-1}\left(R_{0}\right)$ is a smooth one on $S_{3}$. While, in the case where $k=2$, the corresponding point on $S_{3}$ is a quotient singular one. We inspect these in more detail. The quotient map $q_{2}$ can be expressed as follows near the ramification point $R=$ $q_{2}^{-1}\left(R_{0}\right)$. In the case where $k=1$, the point $R$ is nonsingular and there exist open neighborhood $U$ of $R$ with coordinates $(x, y)$ and an automorphism $\sigma_{0}$ satisfying that $\sigma_{0}(x, y)=\left(\omega x, \omega^{-1} y\right)$, where $\omega$ is a primitive cubic root of 1 . Then $q_{2}$ is the quotient map $U \longrightarrow U /\left\langle\sigma_{0}\right\rangle$. On the other hand, in the case where $k=2$, it is easy to see that the point $R$ is a quotient singular point. The morphism $q_{2}$ coincides with the quotient map $U /\left\langle\sigma_{1}{ }^{3}\right\rangle \longrightarrow U /\left\langle\sigma_{1}\right\rangle$, where $\sigma_{1}(x, y)=\left(\zeta x, \zeta^{-1} y\right)$ and $\zeta$ is a primitive 6 -th root of 1 . Therefore $R$ is the singular point of type $A_{1}$. Moreover, the automorphism $\sigma$ in Claim 5 preserves each fiber of $\varphi_{3}: S_{3} \longrightarrow C$, i.e., we have $\varphi_{3} \cdot \sigma=\varphi_{3}$.

Now we consider the singular fibers of $\varphi$. First we treat the case where $P$ is not a Galois point. Since $S_{2}$ is locally defined by $z^{2}-\Psi(t, u, v)=f(u, v)=0$ and $\varphi_{2}$ is given by $\varphi_{2}(z, t, u, v)=(u, v)$, we infer that near a singular point $S_{2}$ can be expressed as $z^{2}=y^{2}+x^{3}$ [resp. $z^{2}=y^{2}+x^{6}$ ] if $P$ is not a 2 -flex [resp. 2-flex]. Looking in the proof of Claim 4, we infer that $\varphi_{2}$ can be given by $\varphi_{2}(x, y, z)=x$. Therefore, if $P$ is not a 2 -flex, the singularity of the fiber is a node. On the contrary, if $P$ is a 2 -flex, then $S_{3}$ has the singularity of type $A_{1}$. Resolving this singularity, we obtain the assertion in the theorem.

Second we treat the case of the singular fiber over a Galois point. If $P=(a, b)$ is a Galois point, then the discriminant $\Psi(t, a, b)$ is a complete square $\prod_{i=1}^{5}\left(t-\alpha_{i}\right)^{2}$. Therefore $\varphi_{2}^{*}(P)$ is locally expressed as $z^{2}-\prod_{i=1}^{5}\left(t-\alpha_{i}\right)^{2}=0$. Hence it is a reducible curve $\Gamma_{1}+\Gamma_{2}$, where $\Gamma_{i} \cong \mathbb{P}^{1}, \Gamma_{1}$ and $\Gamma_{2}$ meet in at five points. Since $\Delta_{i}=q_{2}^{-1}\left(\Gamma_{i}\right)$ is a component of $p_{2}^{-1}(C \times P)$, we infer that $\Delta_{i}$ is isomorphic to $C(i=1,2), \Delta_{1}$ and $\Delta_{2}$ meet in at five points. Moreover at the point corresponding to the 2 -flex $S_{3}$ has an $A_{1}$-singularity. By resolving the singularity, we obtain the singular fiber as in the theorem. Thus we have proved (1), (2) and (3). In the case where $C$ is a general quartic, there exists no 2-flex, hence no Galois point. Therefore the number of 1-flexes is 24, each singular fiber has one node and $S_{3}$ is smooth. Since $p_{2}: S=S_{3} \longrightarrow S_{1}=C \times C$ is a finite double covering, there exists no $(-2)$-curve on $S$. Hence $K_{S}$ is ample. Thus we complete the proof of the theorem. 
Since $\varphi: S \longrightarrow C$ is a semi-stable fibration, we have a holomorphic map $\rho: C \longrightarrow \overline{\mathfrak{H}_{10} / \Gamma_{10}}$, which is the Satake's compactification of the period domain (cf. [1]). Since $\rho$ is not constant, the corollary holds true.

The proof of the assertion of Remark 3.1 is clear. Since $S^{\prime}$ is birational to the minimal splitting variety of the triple covering and is the minimal model, there exists such the isomorphism.

Finally we raise problems.

Problems. (1) Do the similar study as in Theorem 2.1 in the case where $C$ is a smooth curve of degree $d \geq 5$.

(2) Let $C$ be a smooth plane curve of degree $d \geq 3$ and $P$ a point in $\mathbb{P}^{2}$. For the projection $\pi_{P}: C \longrightarrow l$ consider the Galois closure curve $C_{P}$ at $P$. Then we will obtain similarly a smooth threefold $V$ and a morphism $\varphi: V \longrightarrow \mathbb{P}^{2}$, whose fiber over $P$ is isomprphic to $C_{P}$ for a general point $P$. Study the structure of $V$ and singular fibers of $\varphi$. In this case, if $P \in C$, then $\varphi^{*}(P)$ becomes a singular fiber. Are these singular fibers semi-stable, too?

Remark 4. Concerning (2) we have the following remarks.

For all $P \in \mathbb{P}^{2} \backslash C$ the Galois group $G_{P}$ is "constant", i.e., is isomorphic to the full symmetric group of degree $d$ if $C$ is general (cf. [2]). However the moduli of the Galois closure curves will vary. In fact, Sakai [6] has shown that in the case where $C$ is a cubic and the points are general, $C_{P}$ and $C_{Q}$ is not isomorphic to each other if $P$ and $Q$ are close.

\author{
Department of Mathematics \\ FACULTY OF SCIENCE \\ NiIGATA UNIVERSITY \\ NiIGATA 950-2181, JAPAN \\ e-mail: yosihara@math.sc.niigata-u.ac.jp
}

\title{
References
}

[1] W. Barth, C. Peters and A. Van de Ven, Compact Complex Surfaces, Ergebnisse der Mathematik und ihrer Grenzgebiete, 3. Folge Band 4, Springer-Verlag New York, Berlin, Heidelberg and Tokyo, 1984.

[2] F. Cukierman, Monodromy of projections. 15th School of Algebra, Portuguese, Canela, 1998, Mat. Contemp. 16 (1999), 9-30.

[3] S. Iitaka, Algebraic Geometry, Grad. Texts in Math. 76, Springer-Verlag New York, Berlin, Heidelberg and Tokyo, 1982.

[4] K. Miura and H. Yoshihara, Field theory for function fields of plane quartic curves, J. Algebra 226 (2000), 283-294.

[5] M. Namba, Families of Meromorphic Functions on Compact Riemann Surfaces, Lecture Notes in Math. 767, Springer-Verlag Berlin, Heidelberg, New York, 1979. 
[6] H. Sakai, Infinitesimal deformation of Galois covering space and its application to Galois closure curve, preprint.

[7] H. Tokunaga, Triple coverings of algebraic surfaces according to the Cardano formula, J. Math. Kyoto Univ. 31 (1991), 359-375.

[8] H. Yoshihara, Function field theory of plane curves by dual curves, J. Algebra 239 (2001), 340-355.

[9] - Galois points on quartic surfaces, J. Math. Soc. Japan 53 (2001), 731-743. 